\title{
New Partnership for Africa's Development - Nepad
}

\author{
Marcus Röhmer
}

\section{Inhaltsübersicht}

1. Einleitung

2. Entstehung und Teilnehmer

3. Struktur von Nepad

4. Neu: Ein System gegenseitiger Kontrolle

5. Das Verhältnis von Nepad zur AU

6. Ziele von Nepad

7. Das Verhältnis von Nepad zu den G8Staaten

\section{Kritik an Nepad}

\section{Einleitung}

Nepad - das neue Programm für den afrikanischen Kontinent! Ein Marshall-Plan für Aufbau und Entwicklung. ${ }^{1}$ Ein AfrikaAktionsplan, der eine neue, vorher nie dagewesene Partnerschaft mit westlichen Staaten ermöglicht. Ein Bekenntnis $\mathrm{zu}$ mehr Eigenverantwortung, mit dem Verlangen nach mehr Selbständigkeit zugleich. ${ }^{2}$ Oder eben gerade kein Marshall Plan, ${ }^{3}$ mit gar nicht so neuen, dafür aber verbindlich vereinbarten Forderungen? Oder füllte man mit Nepad lediglich alten afrikanischen Wein in neue afrikanische

1 Dominic Johnson/ Martina Schwikowski, Der große Plan schrumpft, in: Tageszeitung vom 26. Juni 2002.

2 Gerhard Schröder, Afrika bekennt sich zu seiner Eigenverantwortung, in: Süddeutsche Zeitung vom 27. Juni 2002.

3 Siehe Fn. 1.
Flaschen um und weiß im übrigen nichts $\mathrm{zu}$ verändern? ${ }^{4}$ In jedem Fall ist es eine Kampfansage gegen die Armut im heutigen Afrika! 5

Wie - das solchermaßen unterschiedlich bewertete - Nepad entstanden und wer daran beteiligt ist, was es beinhaltet und welche Ziele es erfaßt, sowie in welchem Verhältnis es $\mathrm{zu}$ anderen internationalen Organisationen steht, soll in diesem Beitrag beschrieben werden.

\section{Entstehung und Teilnehmer}

Nepad entstand in den Jahren 2000/2001 aus einer Synthese verschiedener afrikanischer Projekte. Das erste Programm „Millienium Partnership for Africa's Recovery Programme "6 wurde von Präsident Mbeki (Südafrika) initiiert. Den Auftrag dazu erhielt er von der (damaligen) Organisation Afrikanischer Einheit (OAU), um gemeinsam mit den Präsidenten Obasanjo (Nigeria) und Bouteflika (Algerien) zu untersuchen, wie die Ursachen der Krise in Afrika zu bewältigen sei. Unterdessen breitete Mbeki schon seine eigene Vision von Afrika, dem "African Renaissance"-Programm aus. Gestützt auf gemeinsame afrikanische Werte wollte er den Anstoß für kulturelle, soziale und politische Reformen in einem sich ver-

$4 \quad$ Maria van Driel, Neuer Wein oder nur alter in neuen afrikanischen Flaschen?, in: Focus-N² von 2002.

5 Bundesministerium für wirtschaftliche $\mathrm{Zu}$ sammenarbeit und Entwicklung, Was ist die Neue Partnerschaft für Afrikas Entwicklung (Nepad)?, 2002.

6 MAP - Millienium Partnership for Africas Recovery Programme. 
einigenden Afrika geben. Dennoch blieb es zu dem damaligen Zeitpunkt beim MAPProgramm, da er sich mit seinen Vorstellungen noch nicht durchsetzen konnte.

Des weiteren besteht Nepad aus dem Omega-Plan. Dieser wurde von Präsident Wade (Senegal) mit starker Unterstützung des Frankophonen Blocks ${ }^{7}$ ins Leben gerufen und angeführt. Das Vorhaben zielte auf den Ausbau der regionalen Infrastruktur und die Förderung von Ausbildungsprojekten.

Als drittes Projekt ist der "Compact for African Recovery" in das Nepad-Programm eingeflossen. Hervorgebracht wurde es von der UN-Economic Commission und galt als Antwort auf einen gemeinsamen Auftrag der afrikanischen Wirtschaftsminister Ende 2000.

$\mathrm{Zu}$ der "New African Initiative" auf einer Ministerkonferenz im Mai 2001 vereinigt, wurde dieses von den Gründungspräsidenten $^{8}$ auf dem G8-Gipfel in Genua vorgestellt.

Im Juli 2001 folgte die Schaffung eines politischen Leitungsorgans. Dieses setzt sich aus 15 Vertretern der Mitgliedstaaten zusammen und ist mit der Leitung des Projektes beauftragt worden. Die Umbenennung in Nepad und die Eröffnung eines Sekretariats in Südafrika nahm der Ausschuß im Oktober 2001 vor. $^{9}$

Die Mitgliedstaaten sind:

- im südlichen Afrika: Mosambik, Botswana, Südafrika;

- in Ostafrika: Mauritius, Ruanda, Äthiopien;

7 Unter Einfluß von Frankreich stehende Staaten.

8 Gründerpräsidenten der Staaten Südafrikas, Nigerias, Senegals, Algeriens und Ägyptens.

9 Alex de Waal, What's new in the 'New Partnership for Africa's Development'?, in: International Affairs, 2002 (78), S. 463 (466).
- in Nordafrika: Algerien, Tunesien, Ägypten;

- in Westafrika: Nigeria, Senegal, Mali;

- in Zentralafrika: Sao Tome und Principe, Gabun, Kamerun.

\section{Struktur von Nepad}

Nepad hat eine vertiefte und intensivere Zusammenarbeit der afrikanischen Staaten zum Ziel. Grundlage dieser Zusammenarbeit soll die Eigenverantwortung und Selbstbestimmung der einzelnen Mitgliedstaaten, aber auch ein System der gegenseitigen Kontrolle sein. Eigens dafür eingerichtete Organe ermöglichen, daß die Politik der Staaten von den Mitgliedstaaten kritisch überprüft und mit Verbesserungsvorschlägen versehen wird.

Organe von Nepad sind der Lenkungsausschuß (steering committee) und das Ausführungskomitee (implementation committee).

Der Lenkungsausschuß besteht aus den Gründerpräsidenten und hat die Leitung, Koordinierung und Weiterentwicklung der Nepad-Programme zum Gegenstand. Dazu gehören die Sicherung und Durchsetzung der Menschenrechte in den Mitgliedstaaten durch die Gewährleistung einer effektiven Kontrolle. Zuständig für die Kontrolle sollen Experten sein, die vom Lenkungsausschuß in allen Bereichen eingesetzt werden. Indikatoren zur Stärkung und Warnsysteme zur frühestmöglichen Erkennung bei der Verletzung von Menschenrechten sollen durch den Ausschuß eingerichtet werden. Außerdem ist er für die Rotation der Tagungsorte unter den Mitgliedern und für Entschuldungsinitiativen auf regionaler Ebene zuständig. Die dadurch erlangten Gelder werden vom Ausschuß für Nepad-Projekte genutzt und somit durch ihn verteilt.

Zudem unterhält er das Nepad-Sekretariat, das in Pretoria von ihm eingerichtet worden ist.

Das Ausführungskomitee besteht aus den Vertretern der 15 Mitgliedstaaten und hat 
bei regelmäßigen Treffen die Aufgabe der gegenseitigen Beobachtung und Einschätzung der einzelnen Staaten. ${ }^{10}$

\section{Neu: System gegenseitiger Kontrolle}

Mit dem neuen Beobachtungs- und Einschätzungsmechanismus rückt Nepad von dem bisherigen System der Nichteinmischung unter Beachtung staatlicher Souveränität ab. Die Konzeption des Kontrollmechanismusses ähnelt der des OECDPrüfungsausschusses für Wirtschafts-, Finanz- und Währungspolitik. Sein Zweck ist es, den Überwachungsmechanismus der Geberländer abzulösen und in eigener Verantwortung die Einhaltung der Ziele von Nepad zu überwachen und gegebenenfalls Empfehlungen auszusprechen. ${ }^{11}$

Damit die Geberländer der Beseitigung ihres Kontrollapparates zustimmen können, müssen ähnliche Kriterien von NepadStaaten akzeptiert und in das neue Überwachungssystem eingebaut werden. Eine Mindestvoraussetzung sollen freie und direkte Wahlen sein. Darüber hinaus sind wirtschaftspolitische Fragen, die Zusammenarbeit der Regierungen beim Verteilen von Hilfsgütern, sowie Inhalt und Umfang der Gesetzgebung und Maßnahmen gegen die Korruption Gegenstand einer ständigen Kontrolle. ${ }^{12}$

Hinter dem System gemeinsamer Kontrolle steht der Gedanke einer engeren Zusammenarbeit unter den Nepad-Staaten. Mit der kontrollierten Verteilung von Hilfsgütern strebt man zum Beispiel an, die Transferkosten der Hilfsgelder gering zu halten. Dazu richtete man einen gemeinsamen Fonds der Geberstaaten ein. Aus diesem können die Nepad-Staaten gemeinsam die eingezahlten Gelder abschöpfen und somit die Kosten vermeiden, die an eine Direkt-

10 Bundesministerium für wirtschaftliche $\mathrm{Zu}$ sammenarbeit und Entwicklung (Fn. 5)

11 Alex de Waal (Fn. 9), S. 471.

12 Alex de Waal (Fn. 9), S. 472. zahlung vom Geber- zum Empfangsstaat gebundenen sind.

Die sich daraus ergebende Ausgrenzung von Staaten, die keine Nepad-Mitglieder sind, versucht man über einen weiteren Fonds aufzulösen. Unklar bleibt, ob die Geberländer bei ungerechter Verteilung zwischen den zwei Fonds ein Kontrollrecht besitzen und damit die Unabhängigkeit der Nepad-Staaten wieder eingeschränkt wird. ${ }^{13}$

\section{Abschied vom strikten Prinzip der Nichteinmischung in die inneren Angelegenheiten?}

Ein weiteres Gebiet der engeren Zusammenarbeit ist die gemeinsame Verpflichtung, den Erwerb von Privateigentum zu erleichtern und an die Nationalität geknüpfte Einschränkungen abzubauen. Daneben treten zahlreiche Friedens- und Sicherheitsinitiativen sowie Ausbauprojekte der Infrastruktur. Mit der zunehmenden Fülle von Aufgaben, mit denen der Ausführungsausschuß betraut ist, geht ein Schwinden an Glaubwürdigkeit in ihn einher. Beispielsweise kritisierten westliche Staaten unter dem Eindruck seiner Zuständigkeit Nepads Verhalten im Anschluß an die Wahlen in Simbabwe. ${ }^{14}$

Nachdem infolge der Wiederwahl von Präsident Mugabe in Simbabwe Folter und Gewalt gegen die Opposition und Journalisten im Lande zunahm, reagierte die EU mit gezielten Sanktionen gegen die Staatsund Parteiführung. Das Commonwealth suspendierte die Mitgliedschaft Simbabwes. ${ }^{15}$ Im Beschlußgremium des Com-

13 Alex de Waal (Fn. 9), S. 474.

14 Alex de Waal (Fn. 9), S. 475.

15 Jule Reimer, Ein Marshallplan für Afrika Die Initiative NePAD auf dem Weltwirtschaftsgipfel, Redaktion: Thomas Weinert, Manuskript vom: 23.Juni $2002 \bullet 18: 40$, Hintergrund Wirtschaft, www.dradio.de/cgibin/es/neu-hintergrundw/196.html. 
monwealths waren auch Mbeki und Obasanjo vertreten. Nachdem sie sich zuerst sträubten, haben sie letztendlich einer Suspendierung von Simbabwe zugestimmt. ${ }^{16}$

Während die westlichen Staaten die zurückhaltenden Stellungnahmen der afrikanischen Staaten kritisierten, warnten sie davor, einen ganzen Kontinent wegen eines Landes zu verurteilen und das Scheitern von Nepad sowie der in diesem Jahr gegründeten $\mathrm{AU}$ von vornherein zu verkünden. Gleichwohl hätte man sich eine etwas deutlicher ausfallendere Reaktion von Nepad und der AU wünschen können. Auf einem der vorbereitenden AUGründungsgipfel fanden die Beeinträchtigungen vor und nach den Wahlen in Simbabwe nicht einmal eine Erwähnung. Dabei schloß man den Präsidentschaftskandidaten von Madagaskar wegen bewaffneter Machtergreifung von eben diesen AUGipfeln aus. ${ }^{17}$ Ein Militärputsch verstoße gegen die AU-Kriterien und führe automatisch zum Ausschluß eines Mitgliedstaates, damit bewiesen die AU-Staaten Konsequenz und Glaubwürdigkeit in der Umsetzung ihrer Ziele.

\section{Nepad und das Verhältnis zur AU}

Die AU ist die Nachfolgeorganisation der OAU. Sie verfolgt das ehrgeizige Ziel, die politische und wirtschaftliche Vereinigung des Kontinents voran $\mathrm{zu}$ bringen und $\mathrm{zu}$ beschleunigen. Damit greift sie, wie auch Nepad, den schon lang existierenden Wunsch nach der Einheit und Unabhängigkeit Afrikas auf. Unter dem Dach der AU sind 17 verschiedene Institutionen geplant, u.a. eine Kommission, ein gemeinsames Parlament, ein afrikanischer Gerichtshof, ein afrikanischer Rat für Wirt-

16 Tony Leon, AU Must Write a New Script for Africa, auf: http://allafrica.com/stories/ 200207120043.html vom 12. Juli 2002.

17 Martina Schikowski, Eine neue Union für ein neues Afrika, in: Tageszeitung vom 9. Juli 2002. schaft, Soziales und Kultur und einige Finanzinstitute. ${ }^{18}$

$$
\begin{gathered}
\text { Wo ist der Platr. } \\
\text { dieses neuen Akteurs? }
\end{gathered}
$$

Verbindungen zwischen Nepad und der AU sind bisher auf informeller Basis gegeben. Der Nepad-Ausführungsausschuß erstattet Berichte an die AU und der AUSekretär ist im Nepad-Lenkungsausschuß auf niedriger Ebene vertreten. ${ }^{19}$ An eine engere Verbindung zwischen beiden Organisationen ist vorerst noch nicht zu denken. Grund für diese geringe Verknüpfung ist zum einem die Dominanz Libyens in der AU. Die Nepad-Staaten befürchten bei Einbindung der AU und auch Libyens ein schwindendes Vertrauen der G8- und OSZE-Staaten in Nepad. ${ }^{20}$ Zum anderen sind die Nepad-Staaten darauf bedacht, ein Aufweichen ihrer Standards zu vermeiden, da bisher jedem afrikanischen Staat die Mitgliedschaft zur AU offensteht. Die von Nepad aufgestellten Kriterien für einen Beitritt sind bei einer engeren Kooperation nur schwer aufrechtzuerhalten.

\section{Ziele von Nepad}

Man bekräftigt immer wieder, Nepad sei nicht nur ein neuer politischer Prozeß zur

18 Alex de Waal (Fn. 9), S. 468.

19 Alex de Waal (Fn. 9), S. 467.

20 Das Verhältnis der G8 - Staaten zu Gaddafi wurde durch dessen Äußerungen, wie „Weiße müßten Afrika generell wieder verlassen, wollten sie bleiben, dann nur als unsere Angestellte", in: Thomas Knemeyer, Von Rhodes bis Mugabe, in: Die Welt vom 22. August 2001, oder „Wir in Afrika brauchen hier nicht viele Parteien", in: Marion Aberle, Sechzig Autos und Waffen für einen kleinen Krieg - Gaddafis Wanderzirkus durch Afrika ist eine Werbetour für seine Vorstellungen einer Afrikanischen Union, in: FAZ vom 16. Juli 2002, sicher nicht verbessert. 
Einwerbung von Gebermitteln für neue Investitionsprogramme. Es stehe vielmehr für politische, soziale und erst zuletzt für wirtschaftliche Reformen.

Hintergrund der Reformziele von Nepad sind neben der gescheiterten Option Entwicklungshilfe und Verschuldung die Einsichten afrikanischer Führer und Präsidenten, für die Probleme Afrikas eine Mitschuld zu tragen. Die Mitgliedstaaten von Nepad erkennen somit an, daß neben der Kolonialherrschaft schlechte Regierungsführung, Demokratiemangel, Mißwirtschaft, Krieg, Gewaltherrschaft und Korruption zu Afrikas Problemen geführt haben. ${ }^{21}$

Daraus ergeben sich im Kern folgende Ziele:

- die Stärkung der Mechanismen für Konfliktprävention auf regionaler und kontinentaler Ebene.

Bisher benannte man die Schlüsselkonflikte Afrikas und einigte sich auf die Fortsetzung bereits bestehender Friedensinitiativen. Weiterhin ist für die Zukunft eine bessere Koordinierung, Reduzierung doppelter und die Verhinderung gegensätzlicher Friedensmissionen geplant. Hierfür verständigte man sich auf die Schaffung eines Friedens- und Sicherheitsrates, bei dem alle Initiativen zusammenlaufen und koordiniert werden können.

Neben den internationalen Bemühungen sollen Strategien zur staatlichen Sicherung des Friedens in den Vordergrund treten. Wesentlich ist dabei die Forderung, staatliche Debatten zu Themen der inneren Sicherheit für die Öffentlichkeit verfolgbar zu gestalten. Neben der Befriedungsfunktion im Land soll dadurch auch das Vertrauen unter den Nachbarstaaten gestärkt, Mißtrauen reduziert und Konflikte vermieden werden. ${ }^{22}$

21 Uschi Eid, Redebeitrag der Parlamentarischen Staatssekretärin im BMZ anläßlich der Afrika-Debatte im Deutschen Bundestag, 25. April 2002.

22 Alex de Waal (Fn. 9), S. 473.
- die Förderung von Demokratie und

- eine verantwortliche Regierungsführung.

Wichtige Kriterien für die Mitgliedschaft bei Nepad und für das System der gegenseitigen Kontrolle sind die Förderung von Demokratie und die Verpflichtung zu einer verantwortlichen Regierungsführung. Voraussetzung für eine effiziente und effektive Regierungsarbeit ist aber auch ein ausreichend institutionalisierter Verwaltungsunterbau. Das Funktionieren der Verwaltung im heutigen Afrika bestimmt sich jedoch maßgeblich aus dem Zusammenspiel geheimer und öffentlicher Verwaltungsarbeit.
Das Funktionieren der Verwaltung
im beutigen Afrika bestimmt sich maßgeblich
aus dem Zusammenspiel geheimer
und öffentlicher Verwaltungsarbeit

Die Bürokratie versteht sich in erster Linie nicht als unparteiisch, gestaltendes Glied, sondern als Verbindung zwischen dem „Big Man“ und seinen Klienten. In der Konsequenz bleibt somit nur ein kleiner Raum für eine effektive und verantwortliche administrative Gewalt. ${ }^{23}$

- eine Kultur der Wahrung der Menschenrechte.

Der Schutz der Menschenrechte soll durch das gegenseitige, afrikanische Einschätzungs- und Überwachungssystem gewährleistet werden.

Weitere politische Ziele sind:

- die Beteiligung der Zivilgesellschaft auf nationaler und sub-nationaler Ebene,

23 Patrick Chabal, The quest for good government and development in Africa: is NEPAD the answer?, in: International Affairs, 2002 (78), S. 447 (451). 
- die Förderung der Partizipation von Frauen,

- die Förderung der Institutionen des Staates,

- die Schaffung rechtsstaatlicher Rahmenbedingungen.

Wirtschaftliche Ziele sind:

- die Einführung einer marktwirtschaftlichen Struktur

- die Hinwendung zu privatem Kapital,

- die Wiederherstellung und Sicherung makro-ökonomischer Stabilität,

- die Schaffung eines transparenten Rahmens für Finanzmärkte sowie für private und öffentliche Rechnungslegung,

- die Förderung von Landwirtschaft und Infrastruktur zur Stärkung einer binnen- und exportorientierten Landwirtschaft und verarbeitenden Industrie,

- die Erweiterung der Kölner Entschuldungsinitiative HIPC II.

Soziale Ziele sind:

- die Stärkung der Bereiche Erziehung und Ausbildung, insbesondere die Steigerung der Schülerzahlen

- der Ausbau und die Verbesserung des Gesundheitswesens mit besonderer Berücksichtigung von $\mathrm{HIV} /$ Aids und Malaria und der signifikanten Verringerung der Kindersterblichkeit.

In Abwägung der Wertigkeit der Zielumsetzung wird kritisiert, dem Aids-Problem käme zu wenig an Bedeutung zu. Es bedrohe die einzelnen Afrikaner, sowie ganze Länder in ihrer Entwicklung; Maßnahmen zur Bekämpfung von Aids sollten demnach im Mittelpunkt von Nepad stehen. ${ }^{24}$

Alex de Waal (Fn. 9), S. 475.

\section{Das Verhältnis von Nepad zu den G8- Staaten}

Effekte infolge der wirtschaftlichen Reformen durch Nepad sollen sein, daß zukünftig mit einem Wirtschaftswachstum von $7 \%$ in den Nepad-Staaten gerechnet werden kann, mit dem Ziel die Armut in Afrika bis $2015 \mathrm{zu}$ halbieren. ${ }^{25}$ Sorgen sollen dafür einerseits Investitionen durch Privatpersonen in allen ökonomischen Bereichen und ein entgegenkommendes Verhalten der G8-Staaten, bei konsequenter Umsetzung der Nepad-Ziele durch deren Mitglieder. Erwartet wird von den G8-Staaten unter anderem die Öffnung ihrer Märkte für afrikanische Produkte und einen Schuldenerlaß für afrikanische Staaten. Schon das Wachstum von 1\% im Export bringe den Nepad-Staaten 70 Milliarden US-Dollar mehr und wirtschaftlich stabilere Verhältnisse mit sich. ${ }^{26}$

Beim letzten G8-Gipfel in Kanada haben die G8-Staaten mit den afrikanischen Staaten und mit Nepad einen Entwicklungspakt geschlossen. Nach diesem Aktionsplan dürfen die Länder mit Investitionen und einem Schuldenerlaß rechnen, die es bereits geschafft haben, vereinbarte Ziele in Nepad zu verwirklichen, oder schon mit

25 Robert Kappel, Die Ursache der Wachstumsschwäche Afrikas - Minimalistische Strategien von Unternehmen, in: Internationales Afrikaforum, 2002 (3), S. 277 (278).

Das von der Weltbank und dem IWF vorausgesetzte Wachstum von $7 \%$ dürfte, angesichts der niedrigen Wachstumsraten in den letzten Jahren schwierig zu erreichen sein. Das BIP wuchs in 12 Ländern um mehr als $5 \%$. Dieselbe Zahl an Ländern hatte Zuwächse zwischen 3 und $5 \%$ zu verzeichnen. In 16 Länder lag das Wachstum zwischen 0 und $3 \%$ und in 6 Länder gab es sogar ein Negativwachstum. Somit konnten lediglich $40 \%$ der Länder Afrikas den durchschnittlichen Wohlstand ihrer Bevölkerung anheben.

26 Karl Müller, Verdeckte Pläne für eine Plünderung Afrikas - G8-Gipfel und Nepad, in: Zeit-Fragen, Nr. 27, Art. 11 vom Juli 2002; Alex de Waal (Fn. 9), S. 470. 
deren Umsetzung begonnen haben. Zudem vereinbarte man allgemein die Aufstokkung der Entwicklungshilfe bis 2006 für Afrika - mit bevorzugter Behandlung derjenigen Staaten, die sich im Sinne westlicher Demokratien entwickeln. Der Entwicklungspakt umfaßt einen Mechanismus der Zusammenarbeit, an dem Nepad beteiligt ist. Jedes der G8-Länder entsendet zu einem bestimmten Thema einen Sonderbeauftragten. Diese treffen sich regelmäßig untereinander und mit ihren afrikanischen Kollegen. Diskutiert werden nur Themen, die zuvor im Konsens unter den G8-Staaten beschlossen wurden. Die aus dem Konsensprinzip folgende Themeneingrenzung wurde von den Nepad-Staaten kritisiert, da eine Ausklammerung von Themen der Ernährungsfragen in Verbindung mit der Gentechnologie wegen des Streits zwischen der EU und USA zu erwarten sei. ${ }^{27}$

\section{Kritik an Nepad}

Gewerkschaften und andere gesellschaftliche Gruppen kritisieren eine mangelhafte Mitwirkungsmöglichkeit an dem Reformprogramm Nepad. Trotz der erklärten zentralen Rolle der Menschen konnten die afrikanischen Völker zu Nepad nichts beitragen. Andere Länder Afrikas stoßen sich an der Vormachtstellung der Gründungsstaaten, da nur ihnen die Besetzung des Lenkungsausschusses zufällt. 36 Länder wurden von der Nepad-Debatte ganz ausgeschlossen. Die Staatsoberhäupter dieser Länder sehen in Nepad ein vom Ausland aufgezwungenes Konzept, wonach sie sich künftig ausrichten müssen und worüber sie aber nicht abstimmen konnten. ${ }^{28}$

Wie sich die Zusammenarbeit innerhalb Nepads entwickeln und welche Rolle der Zusammenschluß in Afrika und im Rahmen der internationalen Beziehungen insgesamt spielen wird, bleibt abzuwarten.

27

Uschi Eid (Fn. 21).

28 Alex de Waal (Fn. 9), S. 475; Jule Reimer (Fn. 15).
Ein „Afrika der zwei Geschwindigkeiten", das mit einem funktionierenden Nepad verbunden sein könnte, birgt Chancen und Risiken. $\mathrm{Ob}$ die möglichen Chancen für die „Fortschrittlicheren" die wahrscheinlichen Risiken für die "Zurückbleibenden" aufwiegen werden, kann angesichts der insgesamt ungünstigen Ausgangssituation Afrikas mit Fug und Recht bezweifelt werden. 


\section{Literaturauswahl:}

\section{Die Organisation stellt sich vor:}

http:/ / www.nepad.org/

http://www.dfa.gov.za/events/nepad.ht $\mathrm{m}$

\section{Die Organisation wird vorgestellt vom BMZ:}

http://www.bmz.de/about/Leitung/afrik abeauftragte/initiative.html

\section{Entwicklungsbericht:}

- African Dialogue II, Promoting Justice and Reconciliation in Africa: Challenges for Human Rights and Development, The Adopted Report of African Dialogue II, 2002.

- Uschi Eid, Afrikas Vision einer selbstbestimmten Entwicklung unterstützen Ansatzpunkte der deutschen Afrikapolitik, 2002.

\section{Weitere Artikel}

Marion Aberle, Sechzig Autos und Waffen für einen kleinen Krieg - Gaddafis Wanderzirkus durch Afrika ist eine Werbetour für sein Vorstellungen einer Afrikanischen Union, in: FAZ vom 16. Juli 2002.

Bundesministerium für wirtschaftliche Zusammenarbeit und Entwicklung, Was ist die Neue Partnerschaft für Afrikas Entwicklung (Nepad)?, 2002.

Partick Chabal, The quest for good government and development in Africa: is NEPAD the answer?, in: International Affairs, 2002 (78).

Maria van Driel, Neuer Wein oder nur alter in neuen afrikanischen Flaschen?, in: Focus - No 2 von 2002.

Uschi Eid, Redebeitrag der Parlamentarischen Staatssekretärin im BMZ anläßlich der Afrika - Debatte im Deutschen Bundestag, 25. April 2002.
Dominic Johnson/ Martina Schwikowski, Der große Plan schrumpft, in: Tageszeitung vom 26. Juni 2002.

Robert Kappel, Die Ursache der Wachstumsschwäche Afrikas - Minimalistische Strategien von Unternehmen, in: Internationales Afrikaforum, 2002 (3).

Thomas Knemeyer, Von Rhodes bis Mugabe, in: Die Welt vom 22. August 2001.

Tony Leon, AU Must Write a New Script for Africa, auf: http://allafrica.com/stories/ 200207120043.html vom 12. Juli 2002.

Karl Müller, Verdeckte Pläne für eine Plünderung Afrikas - G8-Gipfel und Nepad, in: Zeit-Fragen, Nr. 27, Art. 11 vom Juli 2002.

Jule Reimer, Ein Marshallplan für Afrika Die Initiative NEPAD auf dem Weltwirtschaftsgipfel, Redaktion: Thomas Weinert, Manuskript vom: 23. Juni 2002 - 18:40, Hintergrund Wirtschaft, auf: www.dradio.de/cgi-bin/es/neuhintergrundw/196.html.

Martina Schikowski, Eine neue Union für ein neues Afrika, in: Tageszeitung vom 9. Juli 2002.

Gerhard Schröder, Afrika bekennt sich zu seiner Eigenverantwortung, in: Süddeutsche Zeitung vom 27. Juni 2002.

Alex de Waal, What's new in the 'New Partnership for Africa's Development'?, in: International Affairs, 2002 (78). 